PROCEEDINGS OF THE AMERICAN MATHEMATICAL SOCIETY

Volume 125, Number 5, May 1997, Pages 1457-1467

S 0002-9939(97)03731-3

\title{
LIFTING OF KADEC-KLEE PROPERTIES TO SYMMETRIC SPACES OF MEASURABLE OPERATORS
}

\author{
P. G. DODDS, T. K. DODDS, AND F. A. SUKOCHEV \\ (Communicated by Palle E. T. Jorgensen)
}

\begin{abstract}
We show that if $E$ is a separable symmetric Banach function space on the positive half-line, then $E$ has the Kadec-Klee property (respectively, uniform Kadec-Klee property) for local convergence in measure if and only if, for every semifinite von Neumann algebra $(\mathcal{M}, \tau)$, the associated space $E(\mathcal{M}, \tau)$ of $\tau$-measurable operators has the same property.
\end{abstract}

\section{INTRODUCTION AND PRELIMINARIES}

It was shown by J.Arazy [Ar] (see also [Si]) that if a symmetric sequence space $E$ has the Kadec-Klee (or Radon-Riesz) property for pointwise convergence, then the associated unitary matrix space $C_{E}$ has the Kadec-Klee property for the weak operator topology. More recently, the uniform version of this result has been established by Hsu [Hs]. In the present paper, we extend each of these lifting-type results to the setting of symmetric spaces of measurable operators. Our approach is based on a notion of local convergence in measure introduced in [DDDLS]. In the commutative setting, local convergence in measure coincides with the usual notion of convergence in measure on sets of finite measure. On the other hand, for bounded sequences in trace ideals, local convergence in measure coincides with convergence for the weak operator topology. In particular, the results of [Ar], [Hs] for trace ideals follow as special cases of the present approach. We remark that the present paper complements the study of Kadec-Klee properties in non-commutative spaces given in [CDS], [DDDLS], [Su] and effectively reduces the study of such properties in non-commutative spaces to the corresponding question in symmetric spaces of measurable functions on the positive half-line. A systematic study of Kadec-Klee properties in this setting may be found in [CDSS].

We now collect some of the basic facts and notation that will be used in this paper. We denote by $\mathcal{M}$ a semifinite von Neumann algebra with identity 1 on the Hilbert space $\mathcal{H}$, with a fixed faithful and normal semifinite trace $\tau$. If $x$ is a (possibly unbounded) self-adjoint operator in $\mathcal{H}$, we denote by $e^{x}(\cdot)$ the spectral measure of $x$. If $x$ is a closed, densely defined operator in $\mathcal{H}$ such that $u x=x u$ for all unitary $u$ in the commutant $\mathcal{M}^{\prime}$ of $\mathcal{M}$, then $e^{|x|}(B) \in \mathcal{M}$ for all Borel sets

Received by the editors June 2, 1995 and, in revised form, November 27, 1995.

1991 Mathematics Subject Classification. Primary 46L50, 46E30; Secondary 46B20, 47D15.

Key words and phrases. Kadec-Klee properties, rearrangement-invariant spaces, measurable operators, submajorization.

Research supported by A.R.C.

(C)1997 American Mathematical Society 
$B \subseteq[0, \infty)$ and $x$ will be called $\tau$-measurable if and only if there exists $s>0$ such that $\tau\left(e^{|x|}(s, \infty)\right)<\infty$. The collection of all $\tau$-measurable operators is denoted by $\widetilde{\mathcal{M}}$. For $x \in \widetilde{\mathcal{M}}$ and $t \geq 0$, we define the decreasing rearrangement (or generalized singular value function) $\mu(x)$ by setting

$$
\mu_{t}(x)=\inf \left\{s \geq 0: \tau\left(e^{|x|}(s, \infty)\right) \leq t\right\}, \quad t \in[0, \infty) .
$$

For basic properties, we refer to [FK]. If $\epsilon, \delta>0$ then the family of all sets $N_{\epsilon, \delta}$ consisting of all $x \in \widetilde{\mathcal{M}}$ such that $\mu_{\delta}(x)<\epsilon$ form a neighbourhood base at 0 for a Hausdorff linear topology in $\widetilde{\mathcal{M}}$ called the measure topology. Equipped with this measure topology and with sum and product defined as the respective closures of the algebraic sum and product, the space $\widetilde{\mathcal{M}}$ is a complete topological *-algebra.

The measure topology can be localized in the following way [DDDLS]. If $\epsilon, \delta>0$ and if $e$ is a self-adjoint projection in $\mathcal{M}$ with $\tau(e)<\infty$, then the family of all sets $N_{\epsilon, \delta, e}$ consisting of all $x \in \widetilde{\mathcal{M}}$ such that $\mu_{\delta}($ exe $)<\epsilon$ form a neighbourhood base at 0 for a Hausdorff linear topology on $\widetilde{\mathcal{M}}$. This topology will be called the topology of local convergence in measure (denoted $(\mathrm{lcm})$ ). As noted in [DDDLS], convergence locally in measure coincides with convergence for the measure topology relative to $(e \mathcal{M} e, \tau(e \cdot e))$, for each self-adjoint projection $e \in \mathcal{M}$ with $\tau(e)<\infty$. If $\mathcal{M}$ is commutative, and identified with the von Neumann algebra of all multiplication operators given by bounded measurable functions on some localisable measure space then the preceding notion of local convergence in measure reduces to the more familiar notion of convergence in measure on sets of finite measure in the underlying measure space. On the other hand, if $\mathcal{M}$ is the space $\mathcal{L}(H)$ of all bounded linear operators on $H$ and $\tau$ is the standard trace, then for sequences that are bounded in operator norm, convergence locally in measure is precisely convergence for the weak operator topology.

By $L^{0}$ we denote the space of all complex-valued Lebesgue measurable functions on $[0, \infty)$ (with identification a.e. with respect to the Lebesgue measure $m$ ). A Banach space $E \subseteq L^{0}$ is called a rearrangement-invariant Banach function space on $[0, \infty)$ if it follows from $f \in E, g \in L^{0}$ and $\mu(g) \leq \mu(f)$ that $g \in E$ and $\|g\|_{E} \leq\|f\|_{E}$. Furthermore, $E$ is called a symmetric Banach function space if it has the additional property that $f, g \in E$ and $g \prec \prec f$ imply that $\|g\|_{E} \leq\|f\|_{E}$. Here $g \prec \prec f$ denotes submajorization in the sense of Hardy-Littlewood-Polya :

$$
\int_{0}^{t} \mu_{s}(g) d s \leq \int_{0}^{t} \mu_{s}(f) d s, \quad \text { for all } t>0 .
$$

For the general theory of rearrangement-invariant Banach function spaces, we refer the reader to [KPS] and [LT].

Given a semifinite von Neumann algebra $(\mathcal{M}, \tau)$ and a symmetric Banach function space $E$ on $[0, \infty)$ we define the corresponding non-commutative space $E(\mathcal{M}, \tau)$ by setting $E(\mathcal{M}, \tau)=\{x \in \widetilde{\mathcal{M}}: \mu(x) \in E\}$. Equipped with the norm $\|x\|_{E(\mathcal{M}, \tau)}:=$ $\|\mu(x)\|_{E}$, the space $E(\mathcal{M}, \tau)$ is a Banach space and is called the (non-commutative) symmetric operator space associated with $(\mathcal{M}, \tau)$ corresponding to $E$. An extensive discussion of the various properties of such spaces can be found in [DDP1, DDP2]. We shall frequently use the following submajorisation inequality: if $x, y \in \widetilde{\mathcal{M}}$ then

$$
\mu(\mu(x)-\mu(y)) \prec \prec \mu(x-y)
$$


which is proved in [DDP1]. It follows in particular that if $E$ is a symmetric Banach function space on $[0, \infty)$ and if $x, y \in E(\mathcal{M}, \tau)$ then $\|\mu(x)-\mu(y)\|_{E} \leq\|x-y\|_{E(\mathcal{M}, \tau)}$.

Let $X$ be a Banach space and $\mathcal{T}$ a linear space topology on $X$ weaker than the norm topology. The space $X$ is said to have the Kadec-Klee property with respect to $\mathcal{T}$ (denoted $K K(\mathcal{T})$ ) if and only if $x, x_{n} \in X, n=1,2, \ldots, x_{n} \rightarrow x(\mathcal{T})$ and $\left\|x_{n}\right\|_{X} \rightarrow\|x\|_{X}$ imply $\left\|x_{n}-x\right\|_{X} \rightarrow 0$. The space $X$ is said to have the uniform Kadec-Klee property with respect to $\mathcal{T}$ (denoted $U K K(\mathcal{T})$ ) if and only if for every $\epsilon>0$, there exists a $\delta_{X}(\epsilon) \in(0,1)$ such that whenever $x \in X$ and $\left\{x_{n}\right\}$ is a sequence in the unit ball of $X$ with $x_{n} \rightarrow x(\mathcal{T})$ and $\inf _{n \neq m}\left\|x_{n}-x_{m}\right\|_{X} \geq \epsilon$, then it follows that $\|x\|_{X}<1-\delta_{X}(\epsilon)$.

It is straightforward to show (see, for example, [CDSS], Proposition 1.1) that if the symmetric space $E$ on $[0, \infty)$ has $K K(l \mathrm{~cm})$, (a fortiori, if $E$ has $U K K(l \mathrm{~cm})$ ), then $E$ is separable.

\section{The KadeC-KLeE PROPERTy FOR LOCAL CONVERGENCE IN MEASURE}

Let $E$ be a separable rearrangement invariant Banach function space on $[0, \infty)$. Let $(\mathcal{M}, \tau),(\mathcal{N}, \sigma)$ be semifinite von Neumann algebras. We shall need the following result which is [CDS], Lemma 2.3 (see also [Su], Lemma 3).

Lemma 2.1. Let $0 \leq x, y \in \widetilde{\mathcal{M}}$ and $0 \leq u, v \in \widetilde{\mathcal{N}}$. If $x y=0, \mu(x) \prec \prec \mu(u)$ and $\mu(y) \prec \prec \mu(v)$ then $\mu(x+y) \prec \prec \mu(u+v)$.

Lemma 2.2. Assume that $E$ has $K K(\mathrm{lcm})$. For every measurable set $e \subseteq[0, \infty)$ of finite measure, for every $\epsilon>0$, with $0<\epsilon<m(e)$, for every $0 \leq f \in E$ with $\|f\|_{E}=1$ there exists $0<c=c(e, \epsilon, f)<1$ such that whenever $e^{\prime}$ is a measurable subset of $e$ for which $m\left(e^{\prime}\right)=\epsilon$ and $f \geq \epsilon \chi_{e^{\prime}}$ it follows that $\left\|f-\epsilon \chi_{e^{\prime}}\right\|_{E} \leq c$.

Proof. We suppose that the assertion of the Lemma is false. There exist $0 \leq$ $f \in E$ with $\|f\|_{E}=1$, a measurable set $e \subseteq[0, \infty)$ of finite measure, $\epsilon>0$ with $0<\epsilon<m(e)$, and a sequence $\left\{e_{n}\right\}$ of measurable subsets of $e$ such that $m\left(e_{n}\right)=\epsilon, f \geq \epsilon \chi_{e_{n}}, n \geq 1$ and $\left\|f-\epsilon \chi_{e_{n}}\right\|_{E} \rightarrow 1$. Without loss of generality, we may assume $m\left(\bigcup_{n=1}^{\infty} e_{n}\right)=1$, and by composition with a suitable measurepreserving mapping, we may further assume that $\bigcup_{n=1}^{\infty} e_{n}=[0,1]$. Observe that

$$
\begin{aligned}
& \int_{A}\left(f \chi_{[0,1]}-\epsilon \chi_{e_{n}}\right)(s) d s \leq \int_{0}^{t} \mu_{s}\left(f \chi_{[0,1]}\right) d s-\epsilon m\left(A \cap e_{n}\right) \\
& \leq \int_{0}^{t}\left(\mu\left(f \chi_{[0,1]}\right)-\epsilon \chi_{\left[1-m\left(e_{n}\right), 1\right]}\right)(s) d s=\int_{0}^{t} \mu_{s}\left(\mu\left(f \chi_{[0,1]}\right)-\epsilon \chi_{\left[1-m\left(e_{n}\right), 1\right]}\right) d s
\end{aligned}
$$

for all $0<t<1$ and all measurable $A \subseteq[0,1]$ with $m(A)=t$. It follows that

$$
0 \leq f \chi_{[0,1]}-\epsilon \chi_{e_{n}} \prec \prec \mu\left(f \chi_{[0,1]}\right)-\epsilon \chi_{[1-\epsilon, 1]} ;
$$

using Lemma 2.1, we obtain

$$
\begin{aligned}
0 \leq f-\epsilon \chi_{e_{n}} & =f \chi_{[0,1]}-\epsilon \chi_{e_{n}}+f \chi_{[1, \infty)} \\
& \prec \prec \mu\left(f \chi_{[0,1]}\right)-\epsilon \chi_{[1-\epsilon, 1]}+f \chi_{[1, \infty)} \\
& \leq \mu\left(f \chi_{[0,1]}\right)+f \chi_{[1, \infty)}^{\prec \prec f .}
\end{aligned}
$$

It follows that

$$
\left\|\mu\left(f \chi_{[0,1]}\right)-\epsilon \chi_{[1-\epsilon, 1]}+f \chi_{[1, \infty)}\right\|_{E}=1
$$


Without loss of generality, we may assume $f \chi_{[1, \infty)}$ is supported by $\bigcup_{n=1}^{\infty}[2 n, 2 n+1)$. Set $h_{n}=\epsilon \chi_{[2 n-1,2 n-1+\epsilon)}, n \geq 1$ and observe via Lemma 2.1 that

$$
\begin{aligned}
0 \leq \mu\left(f \chi_{[0,1]}\right)-\epsilon \chi_{[1-\epsilon, 1]}+f \chi_{[1, \infty)} & \leq \mu\left(f \chi_{[0,1]}\right)-\epsilon \chi_{[1-\epsilon, 1]}+f \chi_{[1, \infty)}+h_{n} \\
& \prec \prec \mu\left(f \chi_{[0,1]}\right)+f \chi_{[1, \infty)} \prec \prec f .
\end{aligned}
$$

It follows that

$$
\left\|\mu\left(f \chi_{[0,1]}\right)-\epsilon \chi_{[1-\epsilon, 1]}+f \chi_{[1, \infty)}+h_{n}\right\|_{E}=1, \quad n \geq 1 .
$$

Since $h_{n} \rightarrow 0$ locally in measure and since $E$ has $K K(\mathrm{lcm})$, it follows from (2.1) and (2.2) that $\left\|h_{n}\right\|_{E} \rightarrow 0$, and this is clearly a contradiction.

We need the following result for commutative $K K(l \mathrm{~cm})$ spaces.

Proposition 2.3. Assume that $E$ has $K K(l \mathrm{~cm})$. Suppose that $0 \leq f, f_{n}, g_{n} \in E$, for $n=1,2, \ldots$ and that $\left\|g_{n}-f\right\|_{E} \rightarrow 0$. If $0 \leq f_{n} \leq g_{n}$, and if $\left\|f_{n}\right\|_{E} \rightarrow\|f\|_{E}=1$, then $f_{n} \rightarrow f($ lcm $)$ (and hence also $\left\|f_{n}-f\right\|_{E} \rightarrow 0$ ).

Proof. If $f_{n} \nrightarrow f$ locally in measure, then we may assume that there exist $\epsilon>0$, a measurable set $e \subseteq[0, \infty)$ of finite measure and a sequence $\left\{e_{n}\right\}$ of measurable subsets of $e$ such that

$$
m\left(e_{n}\right)>3 \epsilon, \text { and }\left|f_{n}-f\right|>3 \epsilon \chi_{e_{n}}, \quad n \geq 1 .
$$

Since $\left\|g_{n}-f\right\|_{E} \rightarrow 0$, it follows that $\left(g_{n}-f\right) \chi_{e_{n}} \rightarrow 0$ in measure. Consequently, we may assume that there exist measurable subsets $e_{n}^{\prime} \subseteq e_{n}, n \geq 1$, such that $m\left(e_{n}^{\prime}\right)=\epsilon$ and

$$
\left|g_{n}-f\right| \chi_{e_{n}^{\prime}} \leq \epsilon \chi_{e_{n}^{\prime}}, \quad n \geq 1 .
$$

From this it follows that $g_{n}-f_{n} \geq 2 \epsilon \chi_{e_{n}^{\prime}}$ and so

$$
0 \leq f_{n} \leq g_{n}-2 \epsilon \chi_{e_{n}^{\prime}} \leq g_{n}-\epsilon \chi_{e_{n}^{\prime}} \leq g_{n}, \quad n \geq 1 .
$$

Consequently, $\left\|g_{n}-\epsilon \chi_{e_{n}^{\prime}}\right\|_{E} \rightarrow 1$. Now observe that

$$
f-\epsilon \chi_{e_{n}^{\prime}} \geq\left(f \chi_{e_{n}^{\prime}}-\epsilon \chi_{e_{n}^{\prime}}\right)+\left(\left(g_{n}-f\right) \chi_{e_{n}^{\prime}}-\epsilon \chi_{e_{n}^{\prime}}\right)=g_{n} \chi_{e_{n}^{\prime}}-2 \epsilon \chi_{e_{n}^{\prime}} \geq 0, \quad n \geq 1
$$

Further, from

$$
1 \geq\left\|f-\epsilon \chi_{e_{n}^{\prime}}\right\|_{E} \geq\left\|g_{n}-\epsilon \chi_{e_{n}^{\prime}}\right\|_{E}-\left\|g_{n}-f\right\|_{E}
$$

it follows that $\left\|f-\epsilon \chi_{e_{n}^{\prime}}\right\|_{E} \rightarrow 1$. This contradicts the assertion of Lemma 2.2, and suffices to complete the proof.

We need the following estimate, which follows from [CS2], Lemma 2.3 (see also $[\mathrm{Si}])$.

Lemma 2.4. If $E$ is a separable Banach function space on $[0, \infty)$, if $x \in E(\mathcal{M}, \tau)$ and if $e, f$ are self-adjoint projections in $\mathcal{M}$ then

$$
\|\operatorname{exf}\|_{E(\mathcal{M}, \tau)} \leq\|x\|_{E(\mathcal{M}, \tau)}^{\frac{1}{2}}\|f|x| f\|_{E(\mathcal{M}, \tau)}^{\frac{1}{2}} .
$$

Lemma 2.5. Assume that $E$ is separable. If $x, x_{n} \in E(\mathcal{M}, \tau), n=1,2, \ldots$, if $x_{n} \rightarrow x$ (lcm) then there exist a sequence $\left\{e_{n}\right\}$ of self-adjoint projections in $\mathcal{M}$ such that $\tau\left(e_{n}\right)<\infty, n \geq 1$, and a subsequence $\left\{y_{n}\right\} \subseteq\left\{x_{n}\right\}$ such that 
(i) $\left\|e_{n} y_{n} e_{n}-x\right\|_{E(\mathcal{M}, \tau)} \rightarrow 0, \quad\left\|x\left(1-e_{n}\right)\right\|_{E(\mathcal{M}, \tau)} \rightarrow 0, \quad\left\|\left(1-e_{n}\right) x\right\|_{E(\mathcal{M}, \tau)} \rightarrow 0$. If, in addition, $E$ has $K K($ lcm $)$ and $\left\|x_{n}\right\|_{E(\mathcal{M}, \tau)} \rightarrow\|x\|_{E(\mathcal{M}, \tau)}=1$, then the subsequence $\left\{y_{n}\right\}$ given in (i) satisfies

(ii) $\left\|\mu\left(y_{n}\right)-\mu(x)\right\|_{E} \longrightarrow 0$,

(iii) $\left\|\mu\left(e_{n}\left|y_{n}\right| e_{n}\right)-\mu(x)\right\|_{E} \longrightarrow 0, \quad\left\|\mu\left(e_{n}\left|y_{n}^{*}\right| e_{n}\right)-\mu(x)\right\|_{E} \longrightarrow 0$.

Proof. The assertion of (i) follows from [DDDLS], Proposition 2.1.

(ii). Since $\mu\left(e_{n} y_{n} e_{n}\right)-\mu(x) \prec \prec \mu\left(e_{n} y_{n} e_{n}-x\right)$, it follows from (i) that

$$
\left\|\mu\left(e_{n} y_{n} e_{n}\right)-\mu(x)\right\|_{E} \leq\left\|e_{n} y_{n} e_{n}-x\right\|_{E(\mathcal{M}, \tau)} \longrightarrow 0 .
$$

It suffices to show that $\left\|\mu\left(y_{n}\right)-\mu\left(e_{n} y_{n} e_{n}\right)\right\|_{E} \rightarrow 0$. Let $0 \leq z \in E$ be equimeasurable with $\mu(x)$, and let $0 \leq z_{n}, n \geq 1$, be a disjointly supported sequence in $E$ such that $z_{n}$ is disjoint from $z$ and is equimeasurable with $\mu\left(y_{n}\right)-\mu\left(e_{n} y_{n} e_{n}\right)$ for each $n \geq 1$. Observe that $z+z_{n} \rightarrow z(l \mathrm{~cm})$. From Lemma 2.1, it follows that

$$
z+z_{n} \prec \prec \mu(x)+\left(\mu\left(y_{n}\right)-\mu\left(e_{n} y_{n} e_{n}\right)\right)
$$

so that

$$
\left\|z+z_{n}\right\|_{E} \leq\left\|\mu(x)+\left(\mu\left(y_{n}\right)-\mu\left(e_{n} y_{n} e_{n}\right)\right)\right\|_{E} .
$$

Consequently, via (i),

$$
\begin{aligned}
& 1=\|z\|_{E} \leq \underline{\lim }\left\|z+z_{n}\right\|_{E} \leq \varlimsup \lim \left\|z+z_{n}\right\|_{E} \\
& \leq \lim _{n}\left\|\left(\mu(x)-\mu\left(e_{n} y_{n} e_{n}\right)\right)+\mu\left(y_{n}\right)\right\|_{E} \leq \lim _{n}\left\|\mu\left(y_{n}\right)\right\|_{E}=1 .
\end{aligned}
$$

Since $E$ has $K K(l \mathrm{~cm})$, it follows that $\left\|\mu\left(y_{n}\right)-\mu\left(e_{n} y_{n} e_{n}\right)\right\|_{E}=\left\|z_{n}\right\|_{E} \rightarrow 0$, and this suffices to establish (ii).

(iii). From Lemma 2.4, it follows that

$$
\left\|e_{n} y_{n} e_{n}\right\|_{E(\mathcal{M}, \tau)}^{2} \leq\left\|y_{n}\right\|_{E(\mathcal{M}, \tau)}\left\|e_{n}\left|y_{n}\right| e_{n}\right\|_{E(\mathcal{M}, \tau)} \leq\left\|y_{n}\right\|_{E(\mathcal{M}, \tau)}^{2}, \quad n=1,2, \ldots,
$$

and hence from (i),

$$
\left\|\mu\left(e_{n}\left|y_{n}\right| e_{n}\right)\right\|_{E}=\left\|e_{n}\left|y_{n}\right| e_{n}\right\|_{E(\mathcal{M}, \tau)} \longrightarrow 1=\|\mu(x)\|_{E} .
$$

Since $\mu\left(e_{n}\left|y_{n}\right| e_{n}\right) \leq \mu\left(y_{n}\right), n=1,2, \ldots$, it follows from Proposition 2.3 and (ii) that $\left\|\mu\left(e_{n}\left|y_{n}\right| e_{n}\right)-\mu(x)\right\|_{E} \rightarrow 0$, which is the first assertion of (iii). The second assertion of (iii) follows exactly in the same way as the first by observing that $\left\|e_{n} y_{n}^{*} e_{n}-x^{*}\right\|_{E(\mathcal{M}, \tau)} \rightarrow 0$, and that $\mu(x)=\mu\left(x^{*}\right)$.

We can now state the principal result of this section.

Theorem 2.6. If $E$ is a symmetric Banach function space on $[0, \infty)$, then $E$ has $K K(l \mathrm{~cm})$ if and only if $E(\mathcal{M}, \tau)$ has $K K(l \mathrm{~cm})$ for every semifinite von Neumann algebra $(\mathcal{M}, \tau)$.

Proof. We assume that $E$ has $K K(\mathrm{lcm})$ and suppose that $x, x_{n} \in E(\mathcal{M}, \tau), n=$ $1,2, \ldots, x_{n} \rightarrow x(l \mathrm{~cm})$ and $\left\|x_{n}\right\|_{E(\mathcal{M}, \tau)} \rightarrow\|x\|_{E(\mathcal{M}, \tau)}=1$. By Lemma 2.5(i) and passing to a subsequence and relabelling if necessary, we may assume that there exists a sequence $\left\{e_{n}\right\}$ of finite trace projections of $\mathcal{M}$ such that $\left\|e_{n} x_{n} e_{n}-x\right\|_{E(\mathcal{M}, \tau)} \rightarrow 0$. Setting $f_{n}=1-e_{n}, n=1,2, \ldots$, we show first that $\left\|f_{n}\left|x_{n}\right| f_{n}\right\|_{E(\mathcal{M}, \tau)} \rightarrow 0$. Let $A=\bigcup_{n=0}^{\infty}[2 n, 2 n+1), B=\bigcup_{n=0}^{\infty}[2 n+1,2 n+2)$ and let $\phi_{A}: A \rightarrow[0, \infty), \phi_{B}:$ $B \rightarrow[0, \infty)$ be measure preserving bijections. We set

$$
z=\mu(x) \circ \phi_{A}, \quad z_{n}=\mu\left(e_{n}\left|x_{n}\right| e_{n}\right) \circ \phi_{A}, \quad n \geq 1,
$$


and let $0 \leq y_{n}, n \geq 1$, be a disjointly supported sequence in $E$ such that $y_{n} \chi_{A}=0$ and $\mu\left(y_{n}\right)=\mu\left(f_{n}\left|x_{n}\right| f_{n}\right), n \geq 1$. Since $\left\|z-z_{n}\right\|_{E}=\left\|\mu(x)-\mu\left(e_{n}\left|x_{n}\right| e_{n}\right)\right\|_{E}, n \geq 1$, it follows from Lemma 2.5 (iii) that $\left\|z_{n}-z\right\|_{E} \rightarrow 0$ and so also $z_{n} \rightarrow z(\mathrm{lcm})$. Consequently, $y_{n}+z_{n} \rightarrow z(l \mathrm{~cm})$. By Lemma 2.1,

$$
\mu\left(z_{n}+y_{n}\right)=\mu\left(e_{n}\left|x_{n}\right| e_{n}+f_{n}\left|x_{n}\right| f_{n}\right), \quad n \geq 1,
$$

and so

$$
\left\|z_{n}+y_{n}\right\|_{E}=\left\|e_{n}\left|x_{n}\right| e_{n}+f_{n}\left|x_{n}\right| f_{n}\right\|_{E(\mathcal{M}, \tau)}, \quad n \geq 1 .
$$

It follows from Lemma 2.4 and [CKS], Proposition 3.3, that

$$
\begin{aligned}
\left\|e_{n} x_{n} e_{n}\right\|_{E(\mathcal{M}, \tau)}^{2} & \leq\left\|x_{n}\right\|_{E(\mathcal{M}, \tau)}\left\|e_{n}\left|x_{n}\right| e_{n}\right\|_{E(\mathcal{M}, \tau)} \\
& \leq\left\|x_{n}\right\|_{E(\mathcal{M}, \tau)}\left\|e_{n}\left|x_{n}\right| e_{n}+f_{n}\left|x_{n}\right| f_{n}\right\|_{E(\mathcal{M}, \tau)} \leq\left\|x_{n}\right\|_{E(\mathcal{M}, \tau)}^{2}, \quad n \geq 1 .
\end{aligned}
$$

Consequently, it follows from (2.3) that $\left\|z_{n}+y_{n}\right\|_{E} \rightarrow 1=\|z\|_{E}$. Since $E$ has $K K(l \mathrm{~cm})$, it follows that

$$
\left\|f_{n}\left|x_{n}\right| f_{n}\right\|_{E(\mathcal{M}, \tau)}=\left\|\mu\left(f_{n}\left|x_{n}\right| f_{n}\right)\right\|_{E}=\left\|y_{n}\right\|_{E} \longrightarrow 0 .
$$

Since $\left\|x_{n}^{*}\right\|_{E(\mathcal{M}, \tau)}=\left\|x_{n}\right\|_{E(\mathcal{M}, \tau)}$ for all $n$ and $\left\|e_{n} x_{n}^{*} e_{n}-x^{*}\right\|_{E(\mathcal{M}, \tau)} \rightarrow 0$, it follows also that $\left\|f_{n}\left|x_{n}^{*}\right| f_{n}\right\|_{E(\mathcal{M}, \tau)} \rightarrow 0$. From Lemma 2.4, it now follows that

$$
\left\|e_{n} x_{n} f_{n}\right\|_{E(\mathcal{M}, \tau)},\left\|f_{n} x_{n} f_{n}\right\|_{E(\mathcal{M}, \tau)},\left\|f_{n} x_{n} e_{n}\right\|_{E(\mathcal{M}, \tau)} \rightarrow 0 .
$$

That $\left\|x_{n}-x\right\|_{E(\mathcal{M}, \tau)} \rightarrow 0$ is now a consequence of the inequality

$$
\begin{aligned}
\left\|x_{n}-x\right\|_{E(\mathcal{M}, \tau)} \leq & \left\|e_{n} x_{n} e_{n}-x\right\|_{E(\mathcal{M}, \tau)}+\left\|e_{n} x_{n} f_{n}\right\|_{E(\mathcal{M}, \tau)} \\
& +\left\|f_{n} x_{n} e_{n}\right\|_{E(\mathcal{M}, \tau)}+\left\|f_{n} x_{n} f_{n}\right\|_{E(\mathcal{M}, \tau)} .
\end{aligned}
$$

If $E$ is a separable symmetric Banach function space on $[0, \infty)$, then a renorming theorem of A.A. Sedaev [Se] (see also [CDSS], Theorems 1.5, 2.7) shows that there exists an equivalent symmetric norm $\|\cdot\|_{E}^{\prime}$ on $E$ such that $\left(E,\|\cdot\|_{E}^{\prime}\right)$ has $K K(l \mathrm{~cm})$. Theorem 2.6 now implies that $\left(E(\mathcal{M}, \tau),\|\cdot\|_{E(\mathcal{M}, \tau)}^{\prime}\right)$ also has $K K(\mathrm{lcm})$. We now use this observation to give the following criterion for norm convergence, which complements similar criteria given in [CS2].

Corollary 2.7. Let $E$ be a separable symmetric Banach function space on $[0, \infty)$. If $x, x_{n} \in E(\mathcal{M}, \tau), n=1,2, \ldots$, then the following statements are equivalent.

(i) $\left\|x_{n}-x\right\|_{E(\mathcal{M}, \tau)} \rightarrow 0$.

(ii) $x_{n} \rightarrow x\left(\right.$ lcm) and $\left\|\mu\left(x_{n}\right)-\mu(x)\right\|_{E} \rightarrow 0$.

Proof. The implication (i) implies (ii) follows from the inequalities

$$
\left\|\mu\left(x_{n}\right)-\mu(x)\right\|_{E} \leq\left\|x_{n}-x\right\|_{E(\mathcal{M}, \tau)} \quad n \geq 1,
$$

and the fact that norm convergence implies local convergence in measure. To show the implication (ii) implies (i), we may assume, via the above remarks, that $E(\mathcal{M}, \tau)$ has $K K(l \mathrm{~cm})$. Let $x, x_{n} \in E(\mathcal{M}, \tau), n=1,2, \ldots$, and suppose that (ii) holds. It follows immediately that

$$
\left\|x_{n}\right\|_{E(\mathcal{M}, \tau)}=\left\|\mu\left(x_{n}\right)\right\|_{E} \rightarrow\|\mu(x)\|_{E}=\|x\|_{E(\mathcal{M}, \tau)} .
$$

Since $E(\mathcal{M}, \tau)$ has $K K(l \mathrm{~cm})$, it follows that $\left\|x_{n}-x\right\|_{E(\mathcal{M}, \tau)} \rightarrow 0$. 
We remark that the analogues of Theorem 2.6 for the usual Kadec-Klee property (for weak convergence) and for the Kadec-Klee property for convergence in the measure topology have been given in [CDS].

We now turn to a geometric characterisation of the Kadec-Klee property for local convergence in measure, which is based on, and in turn complements, results given in [CDSS]. Suppose that $(E, \leq)$ is a partially ordered normed linear space. The norm $\|\cdot\|_{E}$ is said to be locally uniformly monotone in the sense of Birkhoff if and only if for all $\epsilon>0$ and $0 \leq x \in E$ with $\|x\|_{E}=1$, there exists $\delta_{E}(x, \epsilon)>0$ such that for all $0 \leq y \in E$, whenever $\|x+y\|_{E} \leq 1+\delta_{E}(x, \epsilon)$, it follows that $\|y\|_{E}<\epsilon$. The norm $\|\cdot\|_{E}$ on $E$ is said to be locally uniformly strictly monotone (see [CDSS]) if and only if

$$
x \in E,\left\{x_{n}\right\} \subseteq E, \quad 0 \leq x \leq x_{n}, n=1,2, \ldots,\left\|x_{n}\right\|_{E} \rightarrow\|x\|_{E} \Rightarrow\left\|x_{n}-x\right\|_{E} \rightarrow 0 .
$$

It is a straightforward exercise to show that the norm on $E$ is locally uniformly monotone in the sense of Birkhoff if and only if the norm on $E$ is locally uniformly strictly monotone.

Theorem 2.8. Let $E$ be a symmetric Banach function space on $[0, \infty)$, and consider the following statements.

(i) $E$ has $K K(\mathrm{lcm})$.

(ii) $E(\mathcal{M}, \tau)$ has $K K(l \mathrm{~cm})$, for every semifinite von Neumann algebra $(\mathcal{M}, \tau)$.

(iii) The norm on $E$ is locally uniformly monotone in the sense of Birkhoff.

(iv) The norm on $E(\mathcal{M}, \tau)$ is locally uniformly monotone in the sense of Birkhoff, for every semifinite von Neumann algebra $(\mathcal{M}, \tau)$.

(v) If $x \in E,\left\{x_{n}\right\} \subseteq E$ satisfy $0 \leq \mu(x) \leq \mu\left(x_{n}\right), n=1,2, \ldots$, and if $\left\|x_{n}\right\|_{E} \rightarrow$ $\|x\|_{E}$, then $\left\|\mu\left(x_{n}\right)-\mu(x)\right\|_{E} \rightarrow 0$.

The implications (i) $\Longleftrightarrow$ (ii) $\Rightarrow$ (iii) $\Longleftrightarrow$ (iv) $\Rightarrow$ (v) are always valid. If $E$ is separable, then all statements (i)-(v) are equivalent.

Proof. The equivalence (i) $\Longleftrightarrow$ (ii) is the content of Theorem 2.6. To show the implication (iii) $\Rightarrow($ iv), let $\epsilon>0$ and $0 \leq x \in E(\mathcal{M}, \tau)$ be given. Let $0 \leq f \in E$ be such that $\mu(f)=\mu(x)$ and such that $f$ is supported by $\bigcup_{k=1}^{\infty}[2 k-1,2 k)$. Let $0 \leq y \in E(\mathcal{M}, \tau)$ and suppose that $\|x+y\|_{E(\mathcal{M}, \tau)} \leq 1+\delta_{E}(\epsilon, f)$. Let $0 \leq g \in E$ be such that $\mu(g)=\mu(y)$ and such that $g$ is supported by $\bigcup_{k=0}^{\infty}[2 k, 2 k+1)$. From Lemma 2.1, it follows that

$$
\|f+g\|_{E} \leq\|x+y\|_{E(\mathcal{M}, \tau)} \leq 1+\delta_{E}(\epsilon, f)
$$

and consequently $\|y\|_{E(\mathcal{M}, \tau)}=\|g\|_{E}<\epsilon$, and this suffices to prove the implication (iii) $\Rightarrow($ iv). The implication (iv) $\Rightarrow(v)$ is trivial, and the implication (i) $\Rightarrow$ (iii) follows from [CDSS], Propositions 1.1, 3.2 and Theorem 3.1.

If $E$ is separable, then the implications (v) $\Rightarrow($ iii) $\Rightarrow$ (i) follow from [CDSS], Theorem 3.1 .

Let us mention finally one further consequence of Theorem 2.6. Let $\phi$ be an increasing concave function on $[0, \infty)$ for which $\phi(0)=0=\phi(0+)$. We consider the Lorentz space $\left(\Lambda_{\phi},\|\cdot\|_{\Lambda_{\phi}}\right)$ consisting of all measurable functions $x$ on $[0, \infty)$ for which

$$
\|x\|_{\Lambda_{\phi}}:=\int_{0}^{\alpha} \mu_{s}(x) \phi^{\prime}(s) d s<\infty
$$


The Lorentz space $\Lambda_{\phi}$ is separable if and only if $\lim _{t \rightarrow \infty} \phi(t)=\infty$ and it is shown in [CDSS], Theorem 3.2, that $\Lambda_{\phi}$ is separable if and only if $\Lambda_{\phi}$ has $K K(\mathrm{lcm})$. We therefore obtain the following consequence.

Corollary 2.9. If $\phi$ is a concave real function on $[0, \infty)$ which satisfies $\phi(0)=0=$ $\phi(0+)$ and $\lim _{t \rightarrow \infty} \phi(t)=\infty$, then $\Lambda_{\phi}(\mathcal{M}, \tau)$ has $K K($ lcm $)$ for every semifinite von Neumann algebra $(\mathcal{M}, \tau)$.

For the special case of unitary matrix spaces, we remark that Corollary 2.9 yields a strengthening of [CDS], Corollary 2.11, which is not accessible from the methods of that paper.

\section{Uniform KADEC-KLeE PRoperty FOR LOCAL CONVERGENCE IN MEASURE}

The principal result of this section is the following.

Theorem 3.1. If $E$ is a symmetric Banach function space on $[0, \infty)$, then $E$ has $U K K(l \mathrm{~cm})$ if and only if $E(\mathcal{M}, \tau)$ has $U K K(l \mathrm{~cm})$ for every semifinite von Neumann algebra $(\mathcal{M}, \tau)$.

Proof. We let $\epsilon>0$ and let $x_{n}, n=1,2, \ldots$, be a sequence in the unit ball of $E(\mathcal{M}, \tau)$ such that $x_{n} \rightarrow x \in E(\mathcal{M}, \tau)$ locally in measure and that

$$
\left\|x_{n}-x_{m}\right\|_{E(\mathcal{M}, \tau)}>\epsilon, \quad n \neq m, n, m=1,2, \ldots
$$

By Lemma 2.5(i), we may assume that there exists a sequence $\left\{e_{n}\right\}$ of projections in $\mathcal{M}$ such that $\tau\left(e_{n}\right)<\infty, n \geq 1$ and $\left\|e_{n} x_{n} e_{n}-x\right\|_{E(\mathcal{M}, \tau)} \rightarrow 0$. Since

$$
\left\|\mu\left(e_{n} x_{n} e_{n}\right)-\mu(x)\right\|_{E} \leq\left\|e_{n} x_{n} e_{n}-x\right\|_{E(\mathcal{M}, \tau)}
$$

this implies that $\left\|\mu\left(e_{n} x_{n} e_{n}\right)-\mu(x)\right\|_{E} \rightarrow 0$. Without loss of generality, we may assume that $\left\|x_{n}-x\right\|_{E(\mathcal{M}, \tau)}>\epsilon / 2, \quad n \geq 1$. Setting $f_{n}=1-e_{n}, n \geq 1$, observe that

$$
\begin{aligned}
\left\|x_{n}-x\right\|_{E(\mathcal{M}, \tau)} \leq & \left\|e_{n} x_{n} e_{n}-x\right\|_{E(\mathcal{M}, \tau)}+\left\|e_{n} x_{n} f_{n}\right\|_{E(\mathcal{M}, \tau)} \\
& +\left\|f_{n} x_{n} e_{n}\right\|_{E(\mathcal{M}, \tau)}+\left\|f_{n} x_{n} f_{n}\right\|_{E(\mathcal{M}, \tau)},
\end{aligned}
$$

for all $n \geq 1$. It follows that at least one of the inequalities

$$
\left\|e_{n} x_{n} f_{n}\right\|_{E(\mathcal{M}, \tau)} \geq \frac{\epsilon}{8}, \quad\left\|f_{n} x_{n} e_{n}\right\|_{E(\mathcal{M}, \tau)} \geq \frac{\epsilon}{8}, \quad\left\|f_{n} x_{n} f_{n}\right\|_{E(\mathcal{M}, \tau)} \geq \frac{\epsilon}{8},
$$

holds for infinitely many natural numbers $n$. After suitable relabelling if necessary, we may assume that at least one of these inequalities holds for every $n \geq 1$. We assume first that at least one of the inequalities

$$
\left\|e_{n} x_{n} f_{n}\right\|_{E(\mathcal{M}, \tau)} \geq \frac{\epsilon}{8}, \quad\left\|f_{n} x_{n} f_{n}\right\|_{E(\mathcal{M}, \tau)} \geq \frac{\epsilon}{8}
$$

holds for all $n \geq 1$. In either case, it follows from Lemma 2.4 that $\left\|f_{n}\left|x_{n}\right| f_{n}\right\|_{E(\mathcal{M}, \tau)}$ $\geq \epsilon^{2} / 64, n \geq 1$. We observe that

$$
\mu\left(e_{n} x_{n} e_{n}\right) \leq \mu\left(\left|x_{n}\right| e_{n}\right) \prec \prec \mu\left(\sqrt{\left|x_{n}\right|}\right) \mu\left(\sqrt{\left|x_{n}\right|} e_{n}\right) \leq \frac{1}{2}\left(\mu\left(\left|x_{n}\right|\right)+\mu\left(e_{n}\left|x_{n}\right| e_{n}\right)\right),
$$

and so

$$
\left\|e_{n} x_{n} e_{n}\right\|_{E(\mathcal{M}, \tau)}=\left\|\mu\left(e_{n} x_{n} e_{n}\right)\right\|_{E} \leq \frac{1}{2}+\frac{1}{2}\left\|\mu\left(e_{n}\left|x_{n}\right| e_{n}\right)\right\|_{E}
$$


for all $n \geq 1$. It follows that

$$
\|x\|_{E(\mathcal{M}, \tau)}=\|\mu(x)\|_{E} \leq \frac{1}{2}+\frac{1}{2}\left\|\mu\left(e_{n}\left|x_{n}\right| e_{n}\right)\right\|_{E}+\left\|e_{n} x_{n} e_{n}-x\right\|_{E(\mathcal{M}, \tau)}
$$

for all $n \geq 1$. For each $n=1,2, \ldots$, let $0 \leq z_{n} \in E$ satisfy $\mu\left(z_{n}\right)=\mu\left(e_{n}\left|x_{n}\right| e_{n}\right)$ and let $0 \leq y_{m}^{(n)} \in E, m=1,2, \ldots$, be a disjointly supported sequence with $\mu\left(y_{m}^{(n)}\right)=$ $\mu\left(f_{n}\left|x_{n}\right| f_{n}\right), m=1,2, \ldots$, and such that $z_{n} y_{m}^{(n)}=0, m=1,2, \ldots$ It is clear that $z_{n}+y_{m}^{(n)} \rightarrow_{m} z_{n}(l \mathrm{~cm})$ for each $n$. By Lemmas 2.1, 2.5, for each $n=1,2, \ldots$,

$$
\left\|z_{n}+y_{m}^{(n)}\right\|_{E} \leq\left\|e_{n}\left|x_{n}\right| e_{n}+f_{n}\left|x_{n}\right| f_{n}\right\|_{E(\mathcal{M}, \tau)} \leq 1, \quad m \geq 1 .
$$

Further,

$$
\left\|\left(z_{n}+y_{m}^{(n)}\right)-\left(z_{n}+y_{k}^{(n)}\right)\right\|_{E} \geq\left\|y_{m}^{(n)}\right\|_{E} \geq \frac{\epsilon^{2}}{64}, \quad m \neq k, n \geq 1 .
$$

Using the $U K K(l \mathrm{~cm})$ property of $E$, it now follows that

$$
\left\|z_{n}\right\|_{E}=\left\|e_{n}\left|x_{n}\right| e_{n}\right\|_{E(\mathcal{M}, \tau)}<1-\delta_{E}\left(\frac{\epsilon^{2}}{64}\right), \quad n \geq 1,
$$

and consequently, from (3.1), (3.2) and the fact that $\left\|e_{n} x_{n} e_{n}-x\right\|_{E(\mathcal{M}, \tau)} \rightarrow 0$, we obtain that

$$
\|x\|_{E(\mathcal{M}, \tau)} \leq 1-\frac{1}{2} \delta_{E}\left(\frac{\epsilon^{2}}{64}\right) .
$$

If the inequality $\left\|f_{n} x_{n} e_{n}\right\|_{E(\mathcal{M}, \tau)} \geq \epsilon / 8$ holds for all $n \geq 1$, then we apply the argument of the preceding paragraph to $x^{*}$ and the sequence $\left\{x_{n}^{*}\right\}$. This proves the theorem.

It is possible to characterise the uniform Kadec-Klee property for local convergence in measure in terms of uniform monotonicity properties of the norm on $E$. If $(E, \leq)$ is a partially ordered normed linear space, then the norm $\|\cdot\|_{E}$ is said to be uniformly monotone in the sense of Birkhoff (denoted $U M B$ ) if and only if for all $\epsilon>0$, there exists $\delta_{E}(\epsilon)>0$ such that $0 \leq f, g \in E,\|f\|_{E}=1$ and $\|f+g\|_{E} \leq 1+\delta_{E}(\epsilon)$ imply $\|g\|_{E}<\epsilon$. If, in addition, $E$ is lattice ordered and we suppose that $f \wedge g=0$ in the preceding definition, then the norm on $X$ is said to be disjointly uniformly monotone in the sense of Birkhoff, and this property coincides with property $(C)$ considered in [DDDLS]. It is noted in [Bi], Chap. XV.14, that if $E$ is a Banach lattice with UMB norm, then $E$ is a $K B$-space; that is, every increasing norm-bounded sequence of positive elements of $E$ is convergent. Similarly, if $E$ is a Banach lattice, and if the norm on $E$ is disjointly uniformly monotone in the sense of Birkhoff, then it is noted in [DDDLS] that $E$ satisfies a non-trivial lower estimate. This implies that $E$ contains no lattice copy of $c_{0}$, and again it follows that $E$ is a $K B$-space ([LT], 1.c.4). It now follows from $[\mathrm{Su}]$ that if $E$ is a symmetric Banach function space on $[0, \infty)$, then each of these uniform monotonicity properties is equivalent to the assertion that $E$ has $U K K(\mathrm{lcm})$. Combining these remarks with the result of Theorem 3.1, we obtain the following.

Theorem 3.2. If $E$ is a symmetric Banach function space on $[0, \infty)$, then the following statements are equivalent.

(i) $E$ has $U K K(l \mathrm{~cm})$.

(ii) $E(\mathcal{M}, \tau)$ has $U K K(l \mathrm{~cm})$ for every semifinite von Neumann algebra $(\mathcal{M}, \tau)$.

(iii) The norm on $E$ is $U M B$. 
(iv) The norm on $E(\mathcal{M}, \tau)$ is $U M B$ for every semifinite von Neumann algebra $(\mathcal{M}, \tau)$.

(v) The norm on $E$ is disjointly uniformly monotone in the sense of Birkhoff.

In view of the preceding remarks, the only implication that needs to be established is the implication (iii) $\Rightarrow$ (iv). The details, however, are almost identical to those used to establish the implication (iii) $\Rightarrow$ (iv) of Theorem 2.8, and accordingly are omitted.

As a simple application, we suppose that $\Phi$ is an Orlicz function; that is, $\Phi$ is a continuous convex increasing function on $[0, \infty)$ such that $\Phi(0)=0$ and $\lim _{t \rightarrow \infty} \Phi(t)=\infty$, and let $L^{\Phi}$ be the corresponding Orlicz space equipped with the Luxemburg norm. In this case, it follows from $[\mathrm{AS}]$ that $L^{\Phi}$ has the property $U M B$ if and only if $\Phi$ satisfies the $\Delta_{2}$-condition (i.e. there exist numbers $k$ and $m$ such that $\Phi(2 t) \leq k \Phi(t)$ if $t \geq m)$. We obtain therefore the following consequence of Theorem 3.2.

Corollary 3.3. If $\Phi$ satisfies the $\Delta_{2}$-condition, then $L^{\Phi}(\mathcal{M}, \tau)$ has $U K K(l \mathrm{~cm})$ for every semifinite von Neumann algebra $(\mathcal{M}, \tau)$.

The preceding Corollary is a non-commutative extension of [DV], Proposition 3. Finally, if $E$ satisfies some non-trivial lower estimate (for the precise definition, we refer to $[\mathrm{LT}]$ ), then it is easily seen that the norm on $E$ is disjointly uniformly monotone in the sense of Birkhoff. Accordingly, $E$ and therefore $E(\mathcal{M}, \tau)$ have $U K K(\mathrm{lcm})$. Thus the preceding Theorem 3.1 yields an alternative proof of [DDDLS], Theorem 2.7, at least in the setting of symmetric Banach function spaces rather than in the more general quasi-normed setting considered in [DDDLS].

\section{REFERENCES}

[Ar] J. Arazy, More on convergence in unitary matrix spaces, Proc. Amer. Math. Soc. 83 (1981), 44-48. MR 82f:46009

[AS] M. A. Akcoglu and L. Sucheston, La monotonicité uniforme des normes et théorès ergodiques, C. R. Acad Sc. Paris 301 (1985), 359-360. MR 86k:46037

[Bi] G. Birkhoff, Lattice theory, A. M. S. Colloquium Publications, XXV, 3rd. ed., 1967. MR 37:2638

[CDS] V. I. Chilin, P. G. Dodds and F. A. Sukochev, The Kadec-Klee property in symmetric spaces of measurable operators, Israel J. Math., (to appear). CMP 96:07

[CDSS] V. I. Chilin, P. G. Dodds, F. A. Sukochev and A. A. Sedaev, Characterisations of Kadec-Klee properties in symmetric spaces of measurable functions, 1994. CMP 96:13

[CS1] V. I. Chilin and F. A. Sukochev, Measure convergence in regular non-commutative symmetric spaces, Izv.VUZov (Matematika) 9 (1990), 63-70, (Russian); English translation: Soviet Math., vol. 34, 1990, pp. (78-87). MR 92g:46081

[CS2] V. I. Chilin and F. A. Sukochev, Weak convergence in non-commutative symmetric spaces, J. Operator Theory 31 (1994), 35-65. MR 96e:46085

[CKS] V. I. Chilin, A. V. Krygin and F. A. Sukochev, Extreme points of convex fully symmetric sets of measurable operators, Integr. Equat. Oper. Th. 15 (1992), 186-226. MR 93g:46065

[DDDLS] P. G. Dodds, T. K. Dodds, C. J. Lennard, P. Dowling and F. A. Sukochev, A uniform Kadec-Klee property for symmetric operator spaces, Math. Proc. Camb. Phil. Soc. 118 (1995), 487-502. MR 96h:46034

[DDP1] P. G. Dodds, T. K. Dodds and B. de Pagter, Non-commutative Banach function spaces, Math. Z. 201 (1989), 583-597. MR 90j:46054

[DDP2] P. G. Dodds, T. K. Dodds and B. de Pagter, Non-commutative Köthe duality, Trans. Amer. Math. Soc. 339 (1993), 717-750. MR 94a:46093 
[DV] D. van Dulst and V. de Valk, $(K K)$-properties, normal structure and fixed points of nonexpansive mappings in Orlicz sequence spaces, Can. J. Math. 38 (1986), 728-750. MR 87i:46049

[FK] T. Fack and H. Kosaki, Generalized s-numbers of $\tau$-measurable operators, Pacific J. Math. 123 (1986), 269-300. MR 87h:46122

[Hs] Y. P. Hsu, The lifting of the $U K K$ property from $E$ to $C_{E}$, preprint, 1993. MR 95k:46032

[KPS] S. G. Krein, Ju. I. Petunin and E. M. Semenov, Interpolation of linear operators, Translations of Mathematical Monographs, Amer. Math. Soc. 54 (1982). MR 84j:46103

[LT] J. Lindenstrauss and L. Tzafriri, Classical Banach Spaces II, Springer-Verlag, 1979. MR 81c: 46001

[Se] A. A. Sedaev, On weak and norm convergence in interpolation spaces, Trudy 6 zimney shkoly po mat. programm. i smezn. voprosam. Moscow (1975), 245-267, (Russian). MR 58: 12426

[Si] B. Simon, Convergence in trace ideals, Proc. Amer. Math. Soc. 83 (1981), 39-43. MR 82h: 47042

[Su] F. A. Sukochev, On the uniform Kadec-Klee property with respect to convergence in measure, J. Aust. Math. Soc. (Series A) 59 (1995), 343-352. MR 96h:46022

[Ta] M. Takesaki, Theory of Operator Algebras I, Springer-Verlag, New York-Heidelberg, Berlin, 1979.

Department of Mathematics and Statistics, Flinders University, GPO Box 2100, AdeLAIDE, SA 5001, Australia

E-mail address: peter@ist.flinders.edu.au

E-mail address: theresa@ist.flinders.edu.au

E-mail address: sukochev@ist.flinders.edu.au 